\title{
Orbital Evolution of Dust Particles from Comets and Asteroids
}

\author{
A. A. JACKSON \\ Lockheed Engineering and Sciences Company, Houston, Texas 77258 \\ AND \\ H. A. ZooK \\ NASA Johnson Space Center, Houston, Texas 77058 \\ Received October 30, 1991; revised February 14, 1992
}

In a computer simulation, dust grains of radius 10,30 , and $100 \mu \mathrm{m}$ were released at perihelion passage from each of 35 different celestial bodies: 15 main belt asteroids, 15 short period comets with perihelion greater than $1 \mathrm{AU}$, and 5 short period comets with perihelion less than $1 \mathrm{AU}$. The evolving orbit of each of the 105 released dust grains was then continuously computed with the Everhart numerical integrator until the orbit aphelion passed inside of $0.387 \mathrm{AU}$, or the dust grain had been ejected from the Solar System. The forces due to the gravity of the Sun and the planets as well as radiation pressure, Poynting-Robertson drag, and solar wind drag were all included in these numerical simulations. It is found that when dust grains evolve to intersection with the Earth's orbit, they nearly always retain orbital characteristics indicative of their origins; grains from main belt asteroids differ significantly in orbital characteristics, especially in orbital eccentricity, from grains that evolve from comets. Average intersect velocities with the Earth, before the Earth's gravitational acceleration is taken into account, are about $5 \mathrm{~km} / \mathrm{sec}$ for asteroidal and in excess of $12 \mathrm{~km} /$ sec for cometary-derived grains. Average orbital eccentricities are about 0.1 for asteroidal grains and usually in excess of 0.4 for cometary grains when their orbits can intersect the Earth's orbit. These results mean that accurate trajectory measurements of meteoroids collected with a near-Earth space platform would make it possible to distinguish asteroidal grains from cometary grains. 1992 Academic Press, Inc.

\section{INTRODUCTION}

Whipple (1967) once suggested that Comet Encke quite probably has been the major support for maintaining the quasi-equilibrium of the zodiacal cloud for the past several thousand years. The true contribution of Comet Encke to the meteoritic complex, however, still remains uncertain; indeed, not even the relative contributions of comets and asteroids to the reservoir of interplanetary dust are as yet agreed upon.

The IRAS (Infrared Astronomy Satellite) observations (Low et al. 1984, Hauser et al. 1984), however, have provided important new evidence about the sources of interplanetary dust. Bands of infrared emission were observed parallel to the ecliptic that were interpreted as dust deriving from the collisional breakup of asteroid families (Low et al. 1984, Dermott et al. 1984, Sykes and Greenberg 1986). In addition, dust has been directly observed along the paths of certain comets (Sykes et al. 1986, 1990). Although it may well be true, as Sykes and Greenberg (1986) suggest, that most of the zodiacal emission derives from asteroidal dust grains, it is not at all established that most of the mass of interplanetary dust derives from asteroids. This is true even if it is established that most extraterrestrial grains collected from the stratosphere are of asteroidal origin (Flynn 1989). Relative source strengths remain uncertain, as is also discussed in an excellent review by Leinert and Grün (1990).

Zook $(1986,1988)$ noted that parent body sources of individual dust grains probably could often be determined by accurate measurements of the velocity vectors of those dust grains. That these dust grains should also be captured and returned to terrestrial laboratories for further studies is the purpose of a dust collector and trajectory analyzer that has been proposed for placement into orbit about the Earth (Hörz et al. 1990). Such a facility would not only capture cosmic dust particles with as little destruction as possible but would also determine their state vectors (velocity and position) as they arrive at the detector. The idea being that one uses the orbital data to associate a dust grain with a single parent comet or asteroid, if possible, and then uses 
analytical laboratory analyses of the captured grain to establish the compositional and physical attributes of the parent body. Even if unique parent bodies cannot always be identified, it may be possible to associate a "family" of parent bodies with each captured dust grain.

The evolution of the orbital motion of dust grains when solar radiation and solar gravitational forces alone are acting is well understood (Wyatt and Whipple 1950, Burns et al. 1979). When gravitational forces due to the planets are also included, however, the motion can become quite complex (Gonczi et al. 1982, Burkhardt 1985, Gustafson and Misconi 1986, Gustafson et al. 1987a,b). Not only will there be gravitational scattering by the planets, but the particles may become trapped into one or more orbital mean motion resonances with the planets (Jackson and Zook 1989).

In order to characterize the orbits of particles as they cross the Earth's orbit, a study of the long-term evolution of dust orbital parameters was undertaken. In this paper we display detailed computed time histories of semimajor axis, $a$, eccentricity, $e$, and orbital inclination, $i$, for dust grains started from source bodies in three different, but "typical," types of the originating body orbits. We then present, the orbital eccentricity and velocity-relative-toEarth at Earth orbit nodal crossings for each of three sizes of dust grains derived from 15 different asteroids and 20 different comets. We show that, based on orbital eccentricity alone, particles derived from comets differ significantly from those derived from asteroids when their trajectories cross the Earth's orbit.

\section{COMPUTATIONAL METHOD}

In this study we consider dust particles as spherical ideal blackbodies, of density $1 \mathrm{~g} / \mathrm{cm}^{3}$, with radii from 10 to $100 \mu \mathrm{m}$. Particles of this size are effected by gravitational forces, radiation pressure, Poynting-Robertson (P-R) drag, and solar wind drag (about $30 \%$ of P-R drag). For particles in this size range, radial solar wind pressure and Lorentz $(\mathbf{v} \times \mathbf{B})$ forces are negligible. The gravitational forces of the Sun and Mercury through Jupiter are included for grains from asteroids, and all planets except Pluto for cometary grains; however, the gravitational forces of the planets on each other are always ignored to conserve computational effort, so that they are propagated only in two-body Keplerian orbits about the Sun. The acceleration of the Sun relative to the planets has been ignored.

Our method is to numerically calculate the evolving orbits of individual dust particles after they leave their parent bodies. The motion is determined numerically with the implicit Runge-Kutta integrator of Everhart (1985) using Gauss-Radau spacing. The Sun is at the center of the coordinate system. The acceleration of the dust particle given by

$$
\begin{aligned}
\ddot{\mathbf{r}}=k^{2} \mathbf{r} / r^{3}+ & k^{2} \sum_{i=1}^{N} m \mathbf{r}_{i} / r_{i}^{3}+ \\
& \left(\beta k^{2} / r^{2}\right)[(1-\dot{r} / c) \mathbf{r} / r-(\mathbf{v} / c)(1+s w)],
\end{aligned}
$$

where $\mathbf{r}$ is the distance of the particle from the Sun, $\mathbf{r}_{i}$ is the distance of the particle from the $i$ th planet or mass $m_{i}$, $\mathbf{v}$ is the particle velocity with respect to the Sun, $\dot{\mathbf{r}}$ is its radial velocity with respect to the Sun, $c$ is the speed of light, $k$ is the Gaussian constant, $s w$ is the ratio of solar wind drag to $\mathrm{P}-\mathrm{R}$ drag $(s w=0.3)$, and $\beta$ is the ratio of the gravitational force of the Sun to the radiation pressure force (Burns et al. 1979). $N=5$ for asteroidal grains and $N=8$ for cometary grains.

In each case the particle is assumed to leave the asteroid or comet parent body with zero relative velocity at parent body perihelion. For particles from comets this condition approximates reality. For asteroids this assumption was also made because asteroidal particles so derived would have more highly eccentric and comet-like orbits than if they left their parent asteroid at any other point. As we wish to test whether or not asteroidal grains can be orbitally confused with cometary grains, this gives us something like a "worst case"' test.

We have carried out an analysis of how computed orbital evolution of dust grains depends on the accuracy of the numerical integrator used. According to Everhart (1985), the "global error" of numerical integration using his integrator is less than $10^{-L}$ where " $L$ " is an adjustable parameter that controls the numerical time step size; it does this by requiring that the last term of the "Everhart" expansion sequence be less than $10^{-L}$. Because extensive computer cpu time is required for these runs (a single run of 200,000 years can require an hour of Cray X-MP cpu time), it is important not to require more accuracy or to include more planets than is required to obtain valid results.

For all the calculations shown in this paper, $L=8$ was used. However, we also evaluated the orbital evolution of particles emitted from the 15 main belt asteroids with $L=9,10$, and 11 . These runs were carried out with only the planets Mercury through Jupiter included in two-body Keplerian orbits about the Sun where mutual gravitational interactions between the planets were ignored (as it was for the $L=8$ runs). We found that each time we changed $L$, a different orbital evolutionary sequence ensued for nearly every asteroidal grain. The grains would occasionally capture into new and different orbital period resonances with the Earth or Venus, or not capture at all, when $L$ was changed. Also, close gravitational passes by 
the planets would give different results when different $L$ values were used.

We also tried using the Bulirsch-Stoer integrator (Press et al. 1986) and, again, the details of the orbital evolution differ from the Everhart integrator. Similarly, we have simulated eight planets with asteroidal grains (as was always done for cometary grains), and we ran cases for asteroidal grains with all five planets gravitationally interacting with each other. Again, each different configuration of forces resulted in a different set of detailed dust grain orbital evolutionary histories. We conclude from these analyses that there is a form of "chaos" in these evolutionary histories, where a small change in initial values, in integration accuracy, or in planetary forces applied can result in rather different evolutionary histories. On the other hand, the overall average global history of particle orbit evolution remains the same in the study results we present subsequently. We therefore conclude that-in spite of changes in detail in orbital evolutionary histories caused by neglecting some planets, propagating the planets in two-body Keplerian orbits, or integrator inaccuracies-grains from asteroids remain orbitally distinguishable from cometary grains.

Further information on the accuracy of RADAU integrator may be found in Everhart (1973) and Papp et al. (1977).

\section{INITIAL ORBITS}

Consider a particle ejected from a comet or asteroid due to some outgassing process or due to meteoritic impact. Particles of the radii under consideration will feel the immediate effect of radiation pressure or, the equivalent, a reduced gravitational field of the Sun. Thus the dust particles move on radiation-modified orbits, with new semimajor axes and eccentricities, that differ from their parent comet or asteroid.

Let $a$ and $e$ be the original semimajor axis and eccentricity of the trajectory followed by a particle while it is still part of the parent comet or asteroid. Upon ejection (at zero velocity), the particle will feel solar radiation pressure and move on an orbit with a new semimajor axis $a_{n}$ and eccentricity $e_{\mathrm{n}}$ given by (Kresak 1976)

$$
a_{\mathrm{n}}=(1-\beta)(1-e) a /(1-e-2 \beta)
$$

and

$$
e_{\mathrm{n}}=(e+\beta) /(1-\beta),
$$

where initial release is at perihelion of the orbit.

Figure 1 shows the initial radiation-modified semimajor axis and aphelion for particles of different sizes released from an "average" main belt asteroid (the "average" is taken from Allen (1962)) and for a representative short

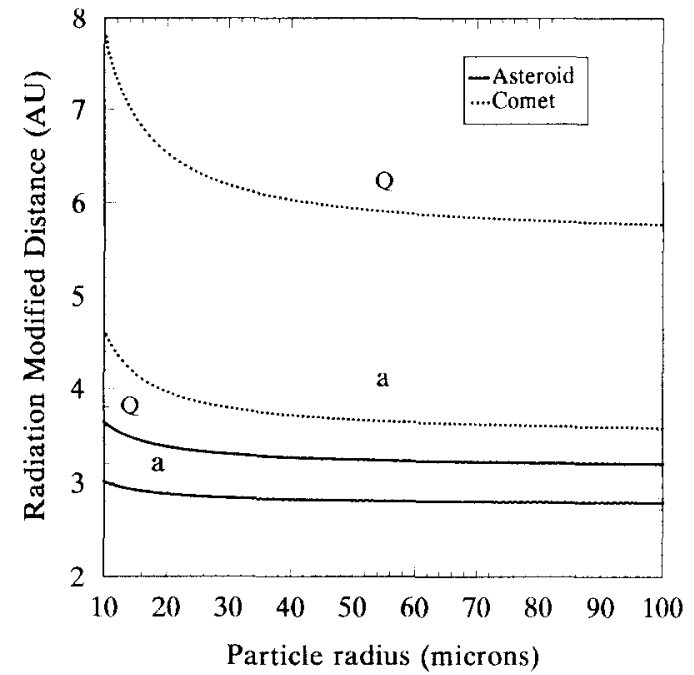

FIG. 1. Photon pressure modification of semimajor axis and aphelion of dust particles upon release from an average asteroid $(a=2.77, e=$ $0.17)$, and a representative short period comet $(a=3.5, e=0.6), a=$ semimajor axis, $e=$ eccentricity, and $Q=$ aphelion distance.

period comet (taken from the range given by Porter (1963)).

We quote these well-known results in order to point out that the dust particles begin their orbital evolution from initial orbits that can differ substantially from those of their parent bodies. Radiation forces can even inject particles directly into orbit-orbit mean motion resonances with a perturbing planet.

The selection of main belt asteroids and their orbital elements are taken from the set of asteroid families of Gradie et al. (1979), and are those archetypical asteroids after which the largest asteroid families were named. They therefore typify, in their orbital characteristics, a very large fraction of all the main belt asteroids. Their average orbital elements are similar to the average of all main belt asteroids (e.g., see Allen 1962). The short period comets and their orbital elements are taken from Marsden (1989). The total list of objects that we selected includes 15 asteroids, 15 comets with perihelia greater than $1 \mathrm{AU}$, and 5 comets with perihelia less than $1 \mathrm{AU}$ and is given in Table I. We particularly concentrated on short period comets with perihelia greater than $1 \mathrm{AU}$ as they typically have lower eccentricities than do Earth-crossing comets; it could be conjectured that dust grains from such comets might well evolve into orbits that are not too different from the evolved orbits of dust grains from asteroids. We shall see that this does occasionally prove to be the case.

\section{ORBIT EVOLUTION}

It might well be surmised that the orbits of dust particles from comets and asteroids would be distinguishable from 
TABLE I

List of Source Bodies with Orbital Elements $(a, e, i)$

\begin{tabular}{lll}
\hline \multicolumn{1}{c}{ Asteroids } & \multicolumn{1}{c}{ Comets $(q>1$ AU) } & \multicolumn{1}{c}{ Comets $(q<1$ AU) } \\
\hline Budrosa $(2.92, .022, .046)$ & Brooks $2(3.63, .490,5.5)$ & Biela $(3.53, .861,12.6)$ \\
Ceres $(2.76, .077,10.6)$ & Clark $(3.12, .502,9.5)$ & Brorsen $(3.1, .81,29.4)$ \\
Concordia $(2.70, .045,5.06)$ & Du Toit-Hartley $(3.36, .602,2.9)$ & Encke $(2.22, .846,11.9)$ \\
Eos $(3.01, .102,10.9)$ & Gunn $(3.60, .316,10.4)$ & Grigg-Skillerup $(2.96, .666,21.1)$ \\
Eunomia $(2.64, .188,11.8)$ & Johnson $(3.64, .367,13.7)$ & Honda-Mrkos-Pajduskova $(2.61, .822,4.2)$ \\
Flora $(2.20, .156,5.89)$ & Kopff $(3.46, .545,4.7)$ & \\
Hertha $(2.43, .151,3.71)$ & Longmore $(3.65, .343,24.4)$ & \\
Hungaria $(1.95, .074,22.5)$ & Neujmin 2 $(3.03, .567,10.6)$ & \\
Koronis $(2.87, .056,1.00)$ & Russell $1(3.34, .517,22.7)$ & \\
Leto $(2.78, .187,7.95)$ & Schwassman-Wachmann $2(3.48, .387,3.7)$ & \\
Maria $(2.55, .065,14.4)$ & Temple $2(3.40, .638,5.4)$ & \\
Nyasa $(2.42, .151,3.7)$ & Temple-Swift $(3.11, .521,10.6)$ & \\
Phocaea $(2.42, .204,2.30)$ & Tuttle-Giacobini-Kresak $(3.15, .502,9.9)$ & \\
Themis $(3.13, .133, .761)$ & Whipple $(3.8, .356,10.2)$ & \\
Undina $(3.21, .082,9.88)$ & Wolf $(4.15, .396,27.3)$ & \\
\hline
\end{tabular}

Note $a$, semimajor axis (AU): $e$, eccentricity; $i$, inclination (deg.).

one another by their orbital eccentricity, inclination, and velocity relative to the Earth, as comets and main belt asteroids largely differ in the first two of these characteristics. However, Poynting-Robertson drag and solar wind drag have the effect of not only causing a dust particle to spiral in toward the Sun but also of reducing the particle's orbital eccentricity (Wyatt and Whipple 1950). Therefore one could imagine that particles evolving from comets with perihelia outside of the Earth's orbit might have their eccentricities reduced enough such that, as they cross the orbit of the Earth, their orbital elements would not be distinguishable from those derived from asteroids.

In addition, gravitational perturbations by the planets could possibly alter the orbits of both asteroidal and cometary particles in such a way as to intermingle their orbits. For asteroidal grains this might happen during passage through a mean motion resonance with Jupiter, Earth, or Mars (Gonczi et al. 1982), possibly while leaving a resonance trap (Jackson and Zook 1989), or due to nearencounter gravitational scattering by a planet. Cometderived dust grains have still more opportunities to experience severe gravitational perturbations as their orbits cross more planets and hence are likely to undergo serious orbital change.

Examples of the variation with time of dust grain orbital parameters $a, e$, and $i$ are shown in Figs. 2 through 10 . After an initial orbit change due to radiation pressure, the particles follow a general decay in semimajor axis due to $\mathrm{P}-\mathrm{R}$ and solar wind drag, except when gravitationally perturbed by the planets.

Figure 2 shows the evolution with time of the semimajor axis of dust grains of 10,30 , and $100 \mu \mathrm{m}$ radius from asteroid Hungaria. The 10- $\mu$ m-radius particle starts with a semimajor axis, modified by radiation pressure, at about 2.1 AU and then rather closely follows a classical P-R and solar wind drag path of decay of semimajor axis. For the $30-\mu \mathrm{m}$-radius particle, the orbital evolution of semimajor axis is similar (though slower in time) except for a small scattering event near Venus. The semimajor axis of the 100- $\mu \mathrm{m}$ grain evolved until it was trapped into a $5: 6$ resonance with the Earth for 140,000 years; in such a trap, the grain orbits the Sun five times for six times for the Earth. After escaping that trap, it was then trapped for a short while near the $1: 1$ resonance with Venus.

One needs to account for the radiation pressure force in locating the mean motion resonance traps. The $j: k$ outer and inner mean motion resonances are located at semimajor axes of

$$
a_{\mathrm{o}}=(1-\beta)^{1 / 3}(k / j)^{2 / 3} a_{\mathrm{p}}
$$

and

$$
a_{\mathrm{i}}=(1-\beta)^{1 / 3}(j / k)^{2 / 3} a_{\mathrm{p}}
$$

where $a_{\mathrm{o}}$ is the outer semimajor axis location, $a_{\mathrm{i}}$ is the inner semimajor axis location, $a_{\mathrm{p}}$ is the perturbing planet's semimajor axis, and $j$ and $k$ are integers such that $k>j$ and, in most cases, jand $k$ differ by one unit.

One sees from the evolution of orbital eccentricity of Hungaria-derived particles in Fig. 3 that, even with gravitational perturbations, orbital eccentricity generally evolves toward zero, but that there are strong variations on that theme. Particularly to be noted, for the $100-\mu \mathrm{m}-$ radius grain, is the very great increase in the orbital eccentricity that occurs starting about 150,000 years after it left asteroid Hungaria. This increase in orbital eccentricity is 

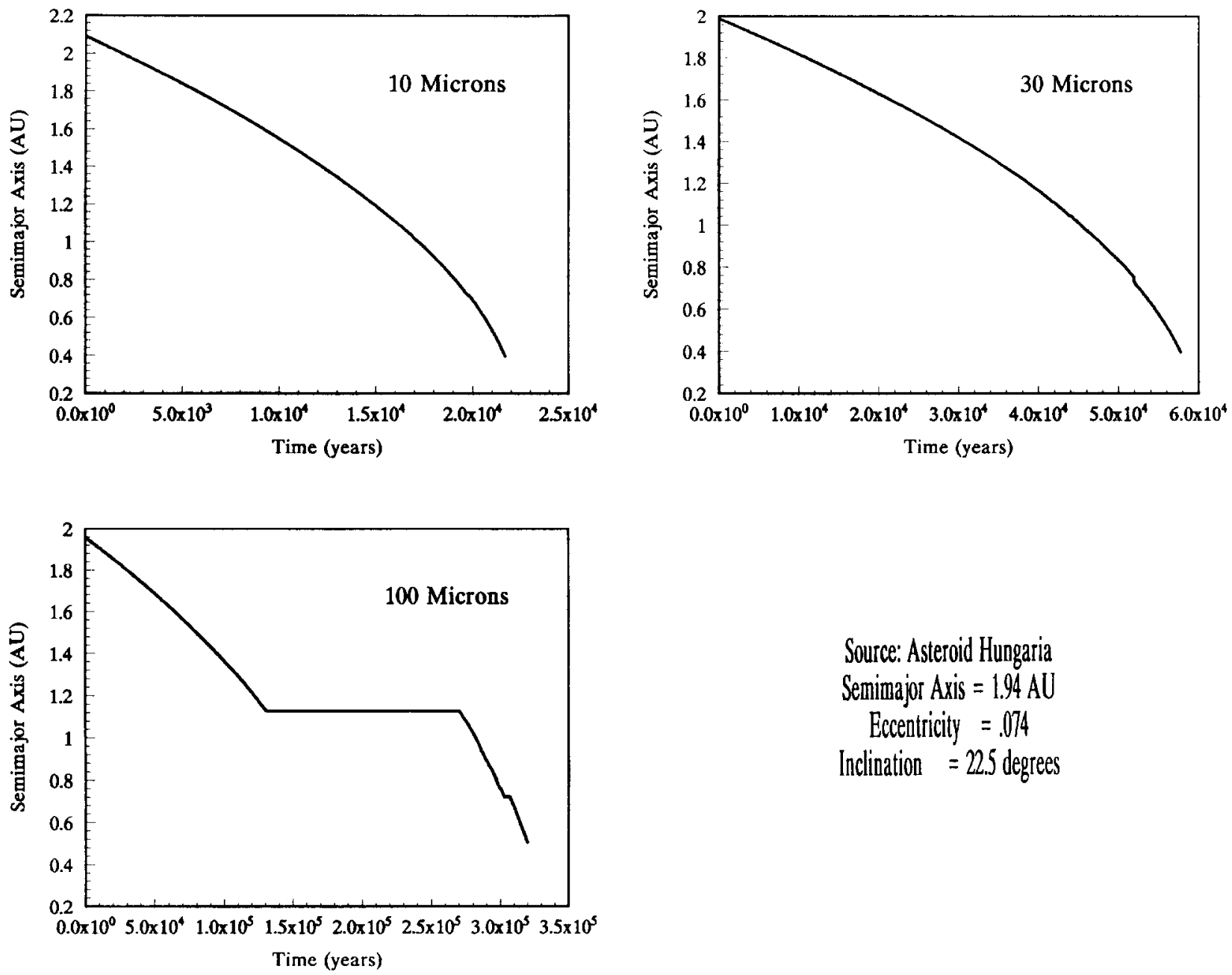

FIG. 2. Semimajor axis evolution of 10-, 30-, and $100-\mu$ m-radius particles originating from asteroid Hungaria.

rather typical during the time that a grain is trapped in an external resonance, as is this grain (see Fig. 2). Also note, however, some decreases in eccentricity during the time it is trapped by the Earth. One also finds that the particle's perihelion passes inside of the Earth's orbit during the trapping interval.

The variation with time of the orbital inclinations of each of the three sizes of dust grains is shown in Fig. 4. Jupiter is responsible for a large proportion of the variation in orbital inclination and eccentricity shown in Figs. 3 and 4, even though the Hungaria-derived particles are never near that planet. Normally, only when a particle is near, or in, a resonance with Earth or Mars, or suffers a close approach, do these planets dominate the orbital evolution.

Dust from comets generally follows much more erratic orbital routes than does dust from asteroids. Figure 5 shows the semimajor axis evolution with time of grains from Comet Temple 2. At 2000 years into its orbital evolution, the $10-\mu \mathrm{m}$-radius particle traps into an interior $8: 5$ resonance with Jupiter, at $a=3.72 \mathrm{AU}$, for over 1000 years. Then it drifts into a very "ragged looking" interior $3: 2$ trap at $3.89 \mathrm{AU}$ about 9500 years into the run for about 2500 years. (The "raggedness" of the trap is due to the fact that periodic close approaches to Jupiter are alternately increasing the semimajor axis above, and decreasing it below, the resonance location at 3.89 AU.) This is not atypical semimajor axis behavior for particles in orbital period traps with the various planets. Because of Jupiter's mass the variation of the semimajor axis is greater than that in traps with other planets. The $10-\mu \mathrm{m}$ grain also remains near a $7: 5$ interior resonance with Jupiter between 12,000 and 22,000 years into the run; it can probably be said to be "trapped" there. Finally, it decays into a 9:5 trap at 36,000 years where it remains for approximately 10,000 years. The particle also experi- 

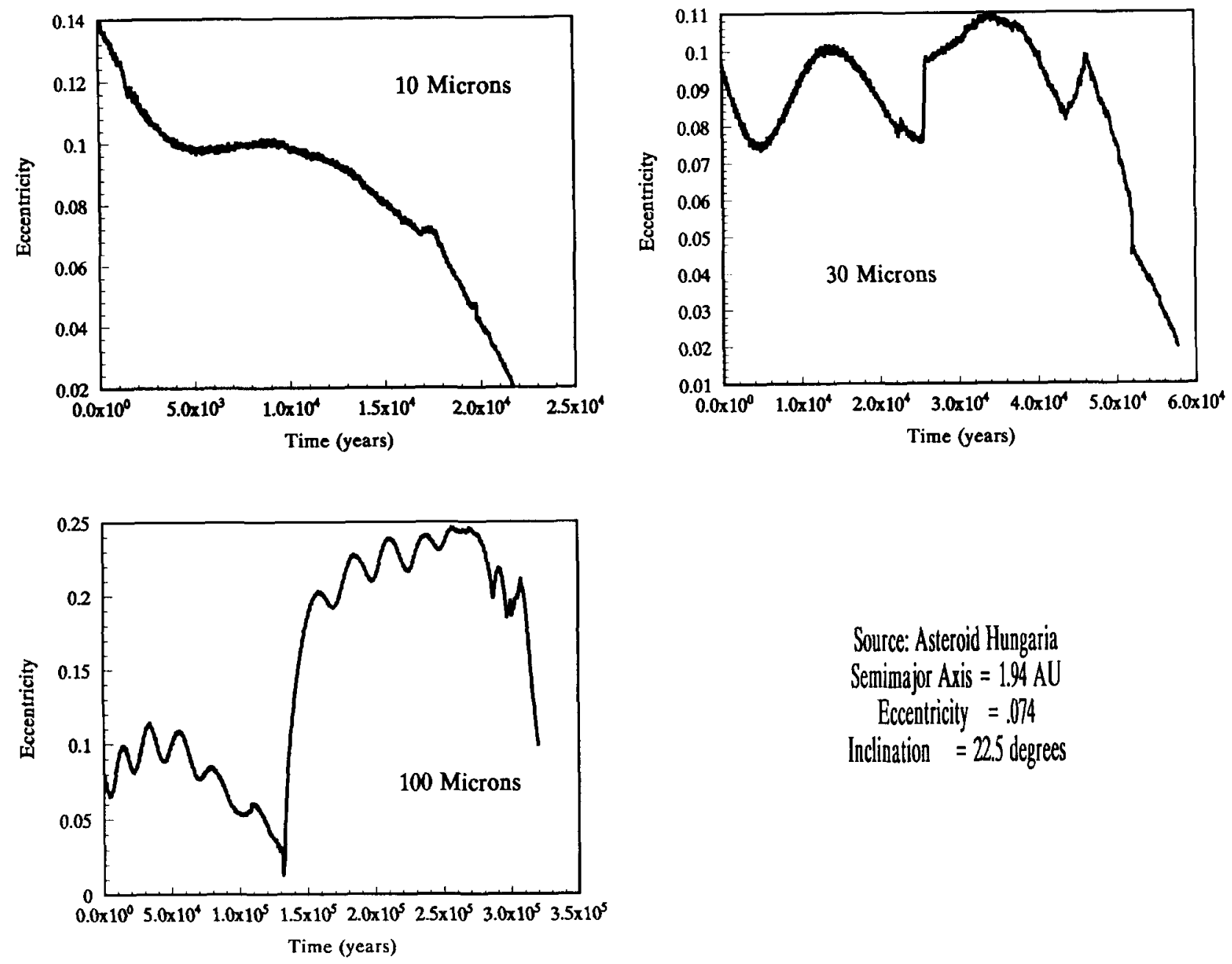

FIG. 3. Orbital eccentricity evolution of 10-, 30-, and 100- $\mu \mathrm{m}$-radius particles originating from asteroid Hungaria.

ences a downward jump in semimajor axis and eccentricity at $3.21 \mathrm{AU}$ as it crosses the interior $2: 1$ resonance with Jupiter during subsequent orbital decay. As may be seen in Fig. 6 for the orbital eccentricity versus time for this particle, the particle's aphelion is usually Jupiter crossing during all but the last of the trapping periods. The complete structure of the somewhat irregular looking traps for the $10-\mu \mathrm{m}$-radius particle in Fig. 5 is not yet completely understood by us and warrants further investigation.

The 30- $\mu$ m-radius grain from Temple 2 was injected directly into a $2: 1$ interior resonance with Jupiter, where it stayed for over 30,000 years, after which it spiraled on in toward the Sun; small downward jogs in the semimajor axis occurred as the particle passed through the $3: 1$ resonance with Jupiter and the $1: 1$ resonance with the Earth. The 100- $\mu \mathrm{m}$ particle from Temple 2 went through a much more complicated orbital history before it was finally ejected from the Solar System after $1.046 \times 10^{6}$ years of orbital evolution. Only the first 730,000 years of its time history are shown in Figs. 5-7. Before the particle became unbound from the Solar System, aphelion was often beyond the orbit of Neptune. It is not at all improbable that a particle of this size would have been destroyed by collison with another meteoroid while undergoing such a long time of orbital evolution (e.g., see Grün et al. (1985) or Leinert and Grün (1990) for collision lifetimes).

The semimajor axis (Fig. 5), eccentricity (Fig. 6), and inclination (Fig. 7) temporal behaviors for dust grains from Comet Temple 2 are not atypical of the behavior of grains from other comets in our ensemble of parent bodies.

Dust grains from comets with perihelia inside of $1 \mathrm{AU}$ evolve quite rapidly, as can specifically be seen for the semimajor axis evolution of dust grains released from Comet Encke in Fig. 8. Except for some minor resonance traps, particles from Encke spiral rather rapidly in toward the Sun. The 10- $\mu \mathrm{m}$-radius grain from Encke was injected 

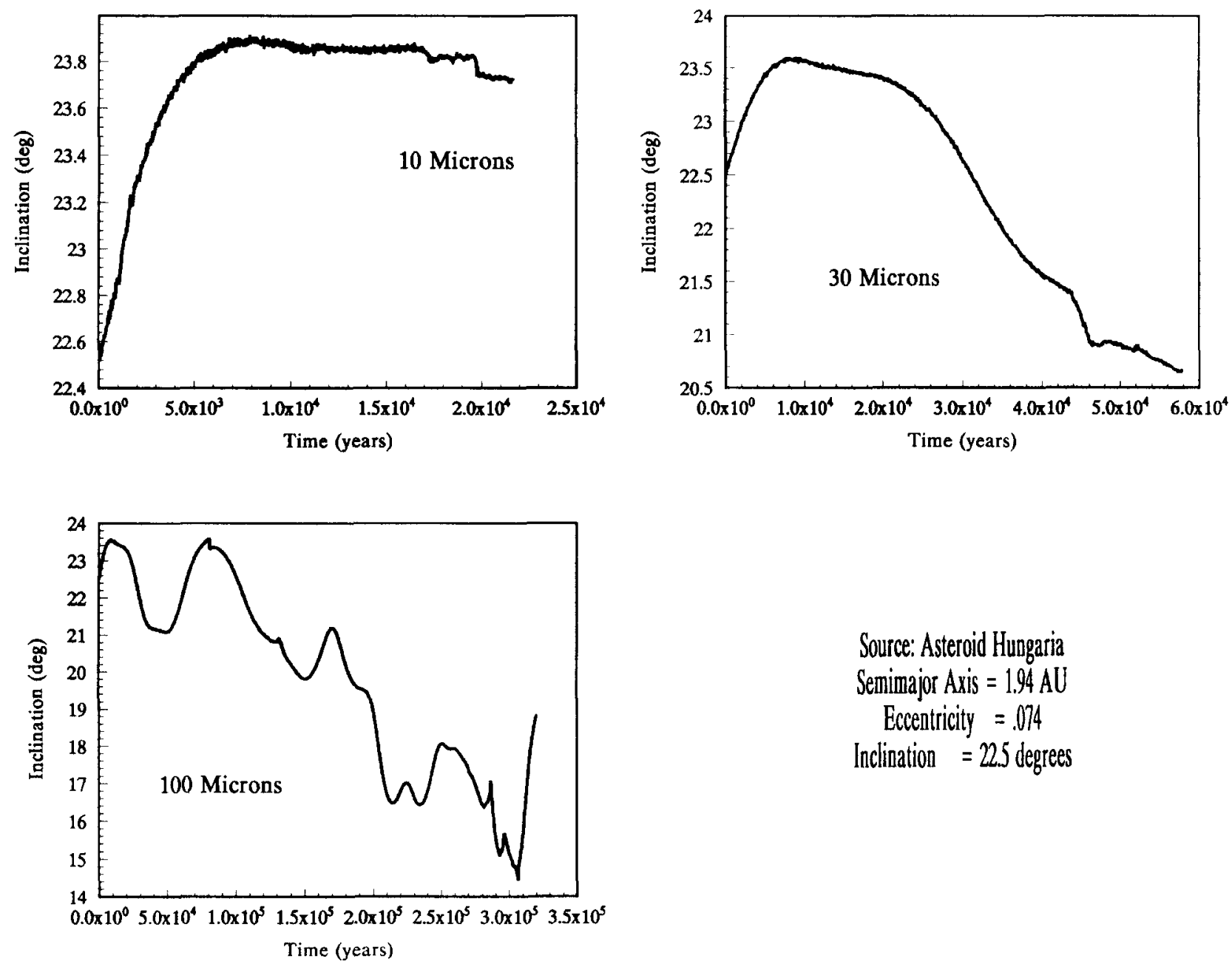

FIG. 4. Orbital inclination evolution of 10-, 30-, and 100- $\mu$ m-radius particles originating from asteroid Hungaria.

into a $1: 2$ external resonance with Jupiter, where it remained trapped for over 1000 years. If the orbital eccentricity of this particle is examined (see Fig. 9), one finds that its initial aphelion was beyond the orbit of Saturn, and that later its aphelion extended beyond the orbit of Uranus for a few thousand years. Although the semimajor axes and eccentricities of the larger grains from this comet varied rather monotonically and smoothly with time, that was not as true for the orbital inclination-as may be noted by examination of Fig. 10.

\section{DUST PARTICLE ORBITS AT 1 AU}

When dust particle orbits evolve to intersect the Earth's orbit, it is not unreasonable to suppose that dust particles originating from comets might well be distinguishable, by their orbital characteristics at that time, from those originating from asteroids.
When the ascending or descending node of a test particle fell within the range 0.983 to $1.017 \mathrm{AU}$ from the Sun, a collision with a body with the Earth was considered possible (neglecting the fact that the longitude of perihelion of the Earth is slowly varying). Various orbital parameters of the dust grains were then examined at the intersection with the Earth's orbit (before acceleration due to terrestrial gravity is accounted for). Two parameters that proved to be especially useful were the orbital eccentricity, $e$, and magnitude of the relative velocity of intersection, $u$.

In Fig. $11, e$ versus $u$ is plotted every 20 years for $10-$ $\mu$-radius dust grains from each of the 35 source bodies during the entire interval that each dust grain node fell inside the range 0.983 to $1.017 \mathrm{AU}$. The circles represent dust grains from asteroids, the squares represent grains from comets with perihelia greater than $1 \mathrm{AU}$, and the triangles represent grains from comets with perihelia less 

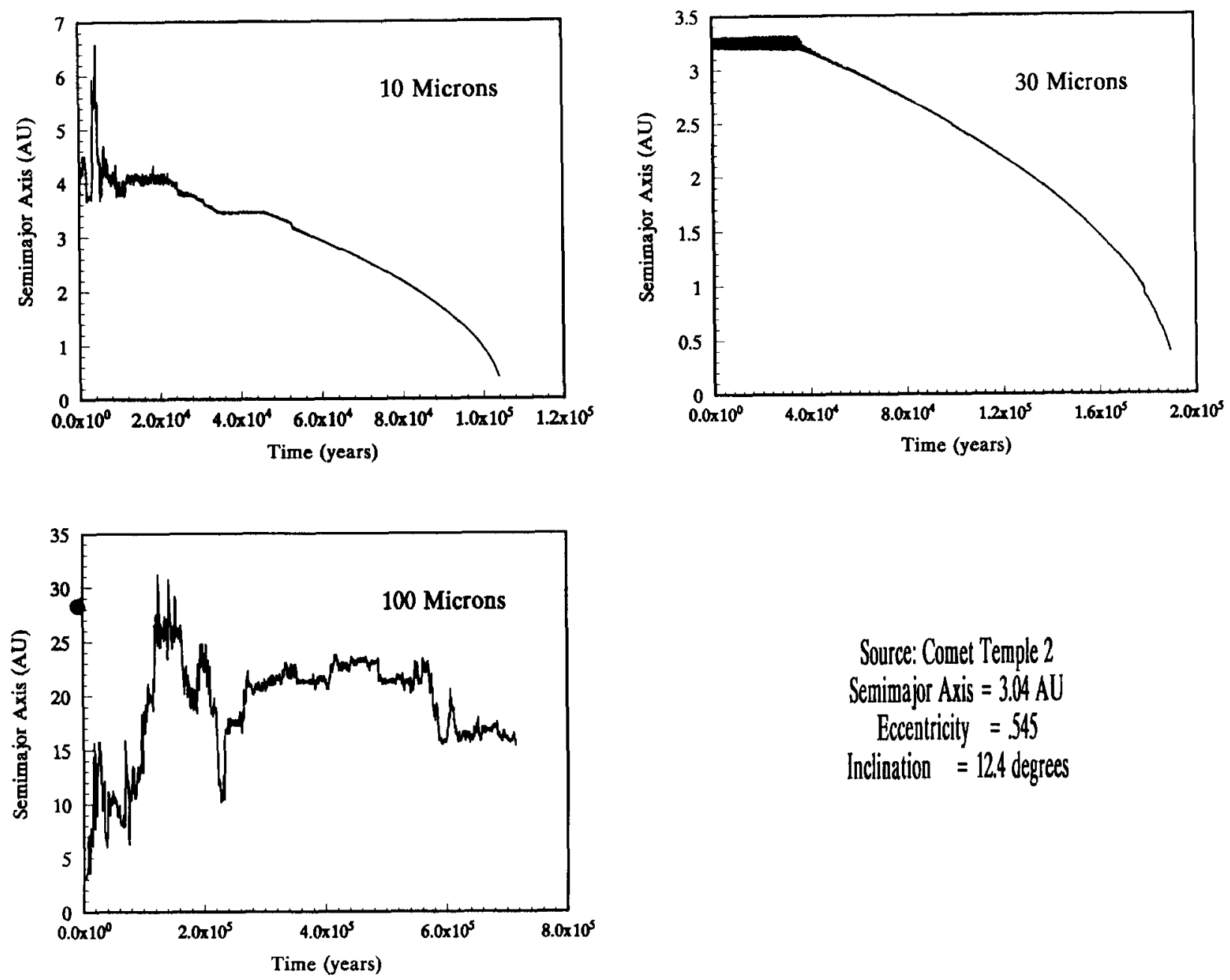

FIG. 5. Semimajor axis evolution of 10-, 30-, and 100- $\mu$ m-radius particles originating from Comet Temple 2.

than 1 AU. Dust grains from Comet Johnson and asteroids Hungaria and Phocaea are specifically identified to aid the reader. Closely spaced groups of symbols usually mean a single dust grain has an ascending or descending node slowly evolving through the range 0.983 to $1.017 \mathrm{AU}$ (taken every 20 years).

Immediately evident in Fig. 11 is the fact that dust grains derived from comets retain high eccentricities (all greater than 0.2 ) while particles from asteroids have low orbital eccentricities. The asteroidal grains also generally have much lower intersect velocities. These results might have been intuitively expected but, due to the gravitational scattering taking place during dust grain orbital evolution, greater confidence could not be gained until detailed evolutionary studies had been carried out, such as the numerical investigation presented here. Although there is some overlap in $u$, there is a clear "gap" in eccentricity values between asteroidal- and cometary-de- rived dust grains of $10 \mu \mathrm{m}$ radius. Grains from comets with perihelia less than $1 \mathrm{AU}$ also seem to be separable from grains originating on comets with perihelia greater than $1 \mathrm{AU}$, although the separation is more gradual.

Figure 12 depicts the same parameters every 50 years for grains of $30 \mu \mathrm{m}$ radius from the same families of parent objects as was done for $10-\mu \mathrm{m}$-radius grains. The same separation in eccentricity occurs, but not with the clear gap, as before. Grains from Comets Temple 2 and TuttleGiacobini-Kresak have $(e, u)$ values that fall into the asteroidal dust grain field. Figure 13 shows the same parameters every 100 years for $100-\mu \mathrm{m}$-radius dust grains. Again, asteroidal grain eccentricities appear, on average, much different than cometary grains, but there is some overlap in their respective $(e, u)$ fields. We note that asteroidal dust grain eccentricities range to somewhat larger values for the larger grains than for the smaller grains. This effect is primarily due to the fact that the larger grains are more 

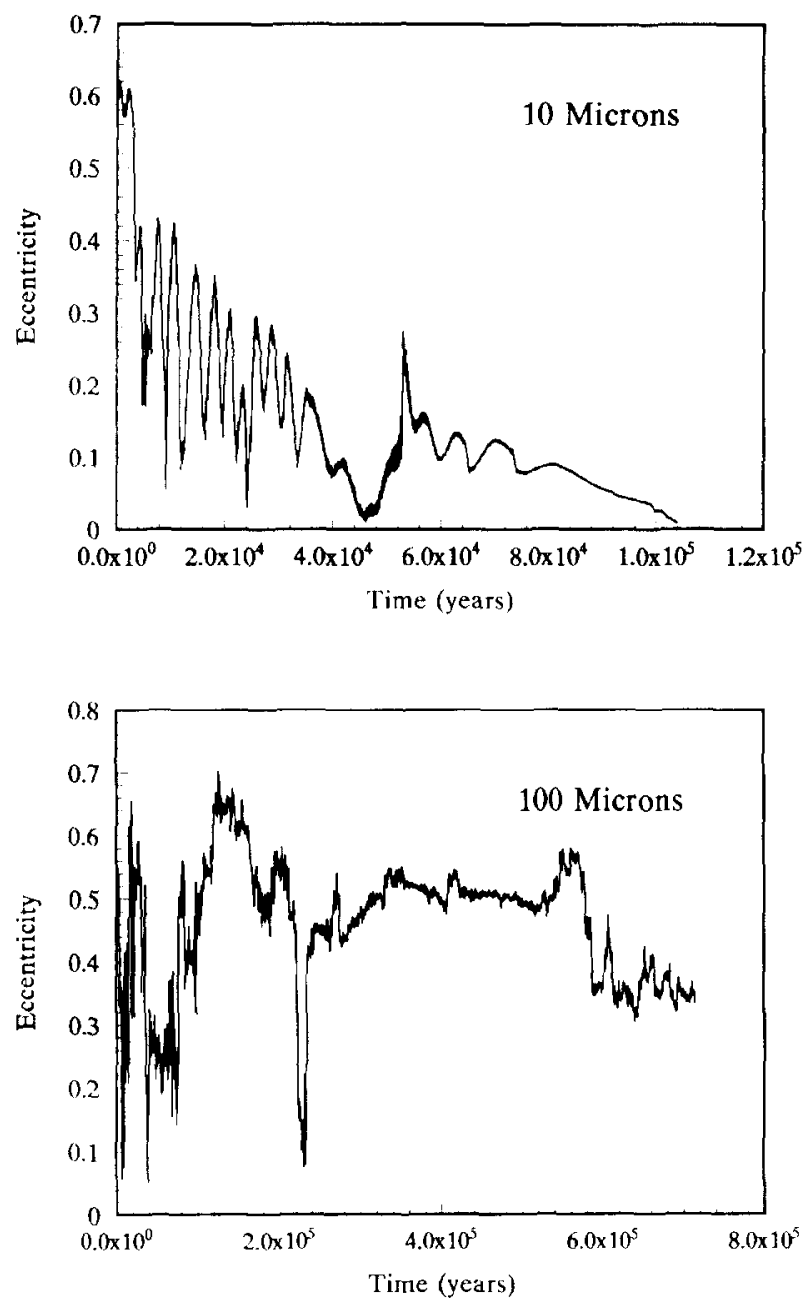

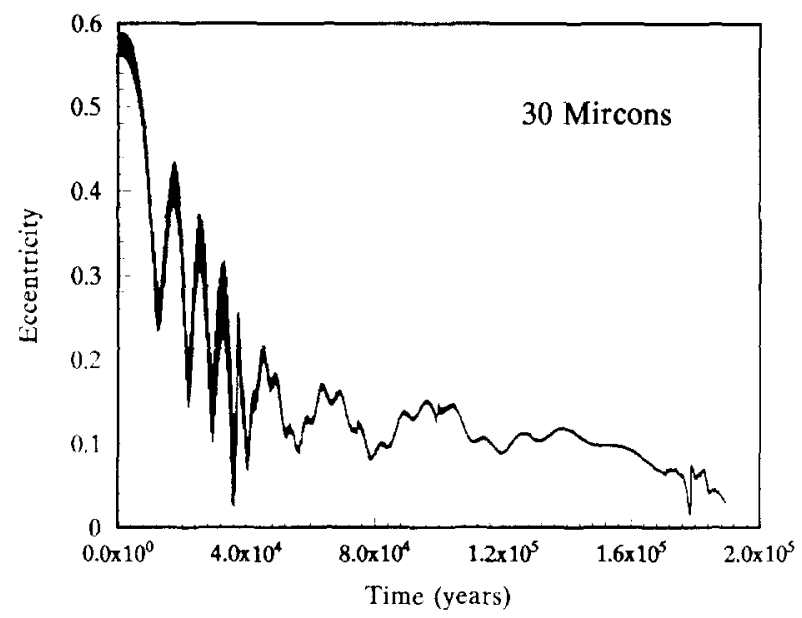

Source: Comet Temple 2

Semimajor Axis $=1.94 \mathrm{AU}$

Eccentricity $=.074$

Inclination $=22.5$ degrees

FIG. 6. Orbital eccentricity evolution of 10-, 30-, and 100- $\mu$ m-radius particles originating from Comet Temple 2.

prone to be trapped into external resonances with the Earth, where their eccentricities grow, before their nodes cross $1 \mathrm{AU}$. This is because the $10-\mu \mathrm{m}$-radius grains evolve so rapidly under $\mathrm{P}-\mathrm{R}$ and solar wind drag forces that trapping by the Earth occurred much less frequently in the orbital simulations of this grain size.

The velocity distributions, at nodal crossing of the Earth's orbit, of dust grains from main belt asteroids are plotted in Fig. 14. These histograms were obtained by summing together in $1-\mathrm{km} / \mathrm{sec}$ "bins" all the $10-\mu \mathrm{m}$ grain velocities obtained in Fig. 11, adding to them all the 30$\mu \mathrm{m}$ grain velocities obtained in Fig. 12, and finally adding to these all the $100-\mu \mathrm{m}$ grain velocities obtained in Fig. 13. The velocity distributions of the three sizes do not differ greatly from each other. This may also be seen from Table II, where mean velocities are given for each dust particle size. Similar velocity distributions are shown in Fig. 15 for dust grains from comets with perihelia greater
TABLE II

Orbital Elements and Velocities at Ascending and Descending Nodal Crossings of the Earth's Orbit

\begin{tabular}{|c|c|c|c|c|c|c|c|c|c|}
\hline & \multicolumn{3}{|c|}{ Asteroids } & \multicolumn{3}{|c|}{ Comets $(q>1 \mathrm{AU})$} & \multicolumn{3}{|c|}{ Comets $(a<1$ AU) } \\
\hline & $10 \mu \mathrm{m}$ & $30 \mu \mathrm{m}$ & $100 \mu \mathrm{m}$ & $10 \mu \mathrm{m}$ & $30 \mu \mathrm{m}$ & $100 \mu \mathrm{m}$ & $10 \mu \mathrm{m}$ & $30 \mu \mathrm{m}$ & $100 \mu \mathrm{m}$ \\
\hline (e) & .095 & .097 & .128 & .472 & .351 & .427 & .723 & .717 & .608 \\
\hline ar & .045 & .022 & .162 & .165 & .153 & .128 & .172 & .077 & .087 \\
\hline$\varepsilon_{\text {nin }}$ & .022 & .015 & .008 & .240 & .015 & .136 & .414 & .477 & .485 \\
\hline$c^{\prime n a x}$ & .162 & .253 & .245 & .740 & .784 & .990 & .973 & .893 & .847 \\
\hline$\langle i\rangle(\mathrm{deg})$ & 7.16 & 6.83 & 9.35 & 10.4 & 21.3 & 15.9 & 17.1 & 16.7 & 14.0 \\
\hline$\sigma$ & 6.61 & 6.06 & 5.90 & 4.79 & 8.96 & 4.88 & 10.8 & 5.20 & 9.50 \\
\hline$i_{\text {min }}$ & 1.47 & .478 & 1.97 & .264 & 5.13 & 6.32 & 1.56 & 5.10 & 2.70 \\
\hline$i_{\text {max }}$ & 24.6 & 23.9 & 24.7 & 40.7 & 41.3 & 42.4 & 46.8 & 33.7 & 46.5 \\
\hline (a) $(\mathrm{km} / \mathrm{sec})$ & 4.79 & 4.89 & 5.92 & 12.1 & 14.5 & 11.8 & 18.4 & 16.4 & 16.4 \\
\hline$\sigma$ & 2.30 & 2.67 & 3.14 & 3.05 & 4.63 & 2.59 & 4.20 & 4.50 & 5.85 \\
\hline$u_{\min }$ & 1.00 & .862 & 1.42 & 5.28 & 7.16 & 7.12 & 11.78 & 10.8 & 10.3 \\
\hline$u_{\mathrm{mix}}$ & 13.45 & 12.56 & 12.7 & 25.3 & 26.7 & 28.4 & 32.4 & 32.1 & 29.0 \\
\hline
\end{tabular}

Note. $\langle e\rangle$ is the average eccentricity, or is its standard deviation, $e_{\text {min }}$ is its minmum, and $\ell^{\prime}$ nita is its maximum. Similarly for the inclination, $i$, and the magnitude of the relative velocity. 11 , at crossing. 

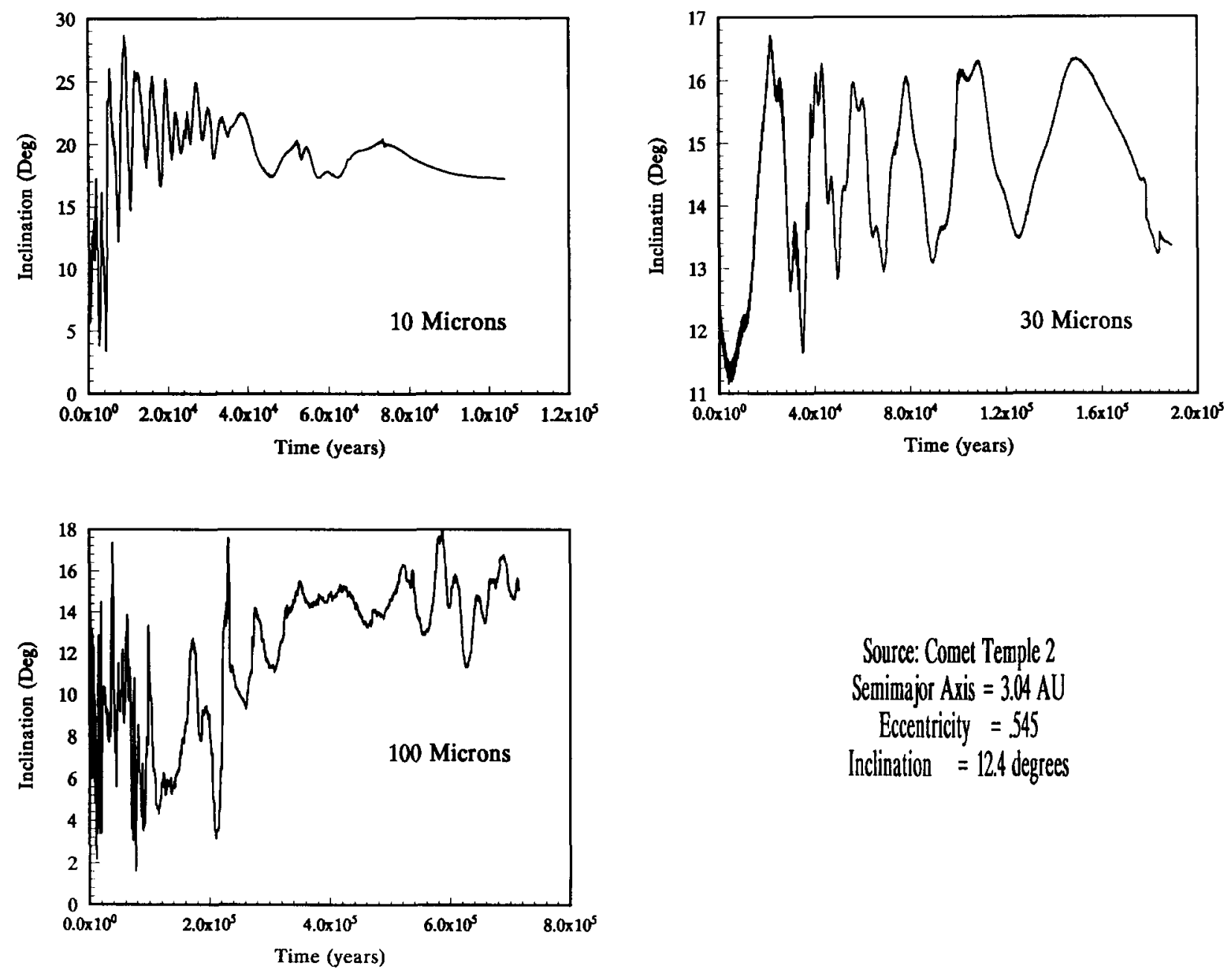

FIG. 7. Orbital inclination evolution of 10-, 30-, and 100- $\mu$ m-radius particles originating from Comet Temple 2.

than $1 \mathrm{AU}$ and in Fig. 16 for dust grains from comets with perihelia less than $1 \mathrm{AU}$. Dust from comets with perihelia greater than $1 \mathrm{AU}$ have nodal intersect velocities that are generally intermediate between those of dust from asteroids and those of dust from comets with perihelia less than $1 \mathrm{AU}$. Entry velocities of dust grains into the Earth's atmosphere are obtained from these histograms by accounting for the gravitational acceleration and focusing to the top of the atmosphere.

Averages, standard deviations, maximums, and minimums of dust grain orbital eccentricity, $e$, inclination, $i$, and intersect velocity, $u$, are given in Table II for each of three sizes of dust grains from asteroids, from comets with perihelion greater than $1 \mathrm{AU}$, and from comets with perihelion less than $1 \mathrm{AU}$. These are taken during the interval that the dust grain orbits can intersect the Earth's orbit. Dust grains from each of the three source body families are seen to differ significantly, on average, from each other in the parameters $e, i$, and $u$.

\section{DISCUSSION}

Grains of $10 \mu \mathrm{m}$ radius released from asteroids are very infrequently trapped into resonance with some planet as their orbits evolve inward toward the Sun, while $10-\mu \mathrm{m}$ radius grains from comets are quite often trapped into both interior and exterior resonances with Jupiter. Larger asteroidal grains are occasionally injected directly into an interior resonance trap with Jupiter, but they almost never drift into such a resonance. The difficulty of drifting, and trapping, into an interior resonance has already been noted by Gonzci et al. (1982). But we note here that this difficulty seems to apply only when the released particle's aphelion does not cross the planet. 

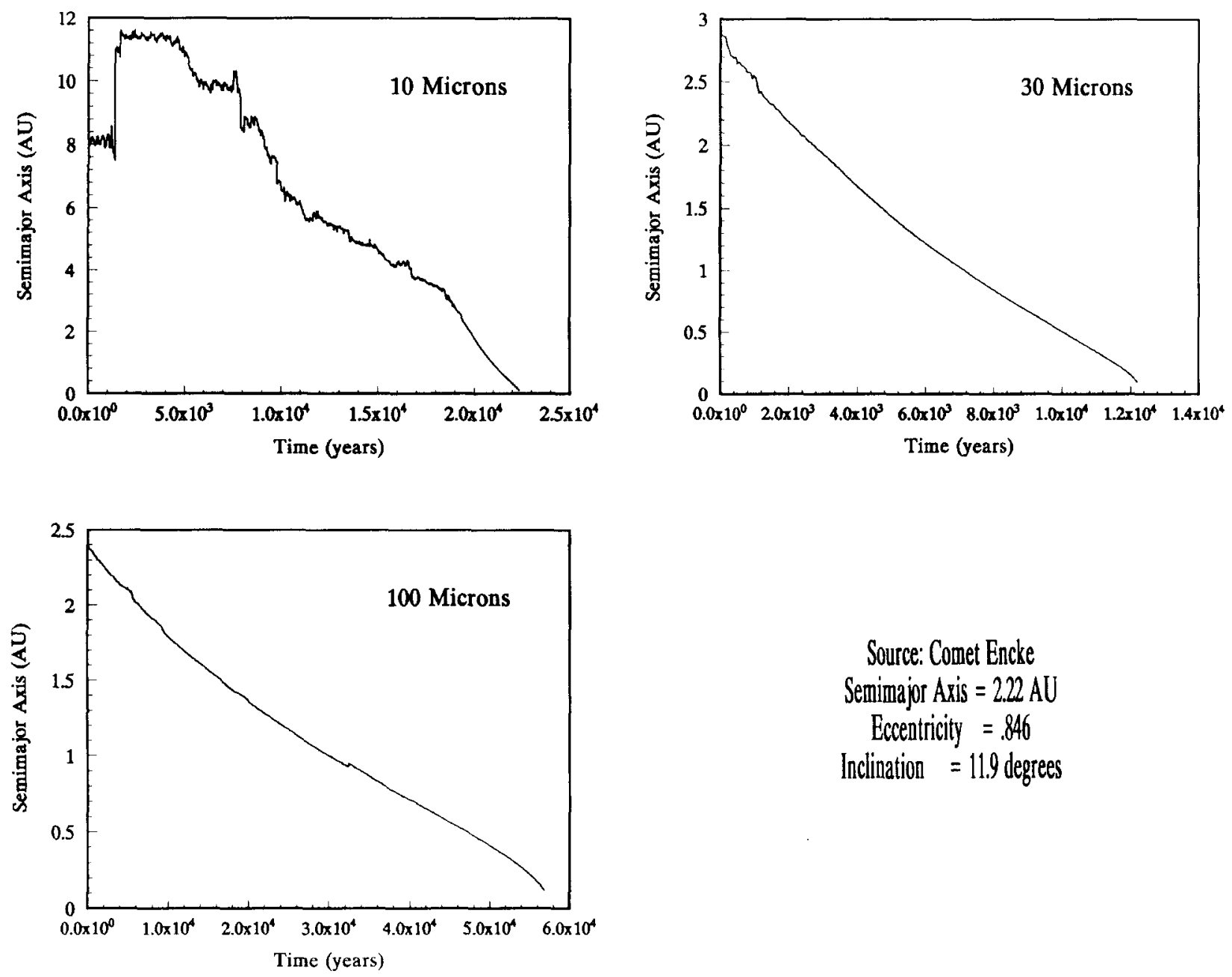

FIG. 8. Semimajor axis evolution of 10-, 30-, and 100- $\mu \mathrm{m}$-radius particles originating from Comet Encke.

For asteroidal grains of $30 \mu \mathrm{m}$ radius, only about half were trapped into external resonances with the Earth as they evolved sunward, and the average time they remained in the trap, when they were trapped, was about 15,000 years. This was about half of the value that had been earlier found (Jackson and Zook 1989), and is probably due to the additional planets simulated as well as to different initial conditions. Like Gonzci et al. (1982), we note that when particles are not trapped as they orbitally evolve through a resonance location they often make abrupt decreases in semimajor axis during such a passage.

We now comment again on why we chose the parent bodies that we did and on why we assumed all dust grains were released with zero velocity at perihelion of the parent body. First, we wanted the asteroidal dust grains to represent the dust grains that would most likely be produced in the main belt of asteroids; the 15 major asteroid families given by Gradie et al. (1979) appear to represent the main belt rather well in terms of the spread of orbital parameters and in terms of averages of orbital parameters. Second, the dust grains were released at perihelion because, for cometary grains, that best simulates their origin; for asteroidal grains this release point was chosen because it maximizes the eccentricity of the released grain (due to radiation pressure) and should more sharply test whether or not asteroidal grains can be orbitally differentiated from cometary grains after they have orbitally evolved. Third, we mostly chose short period comets with perihelion greater than $1 \mathrm{AU}$ for evaluation, not because they are the most copious grain emitters, but because grains from such comets would have lower initial eccentricity values than grains from other comets and might most easily be confused with grains from asteroids. Such a choice of parent comets provides a good test of whether or not asteroidal grains can be differentiated from nearly all cometary grains. In fact, as we see in Figs. 11-13, some grains from these comets do have $(e, u)$ characteristics similar to those of grains from some asteroids. One is thus encour- 

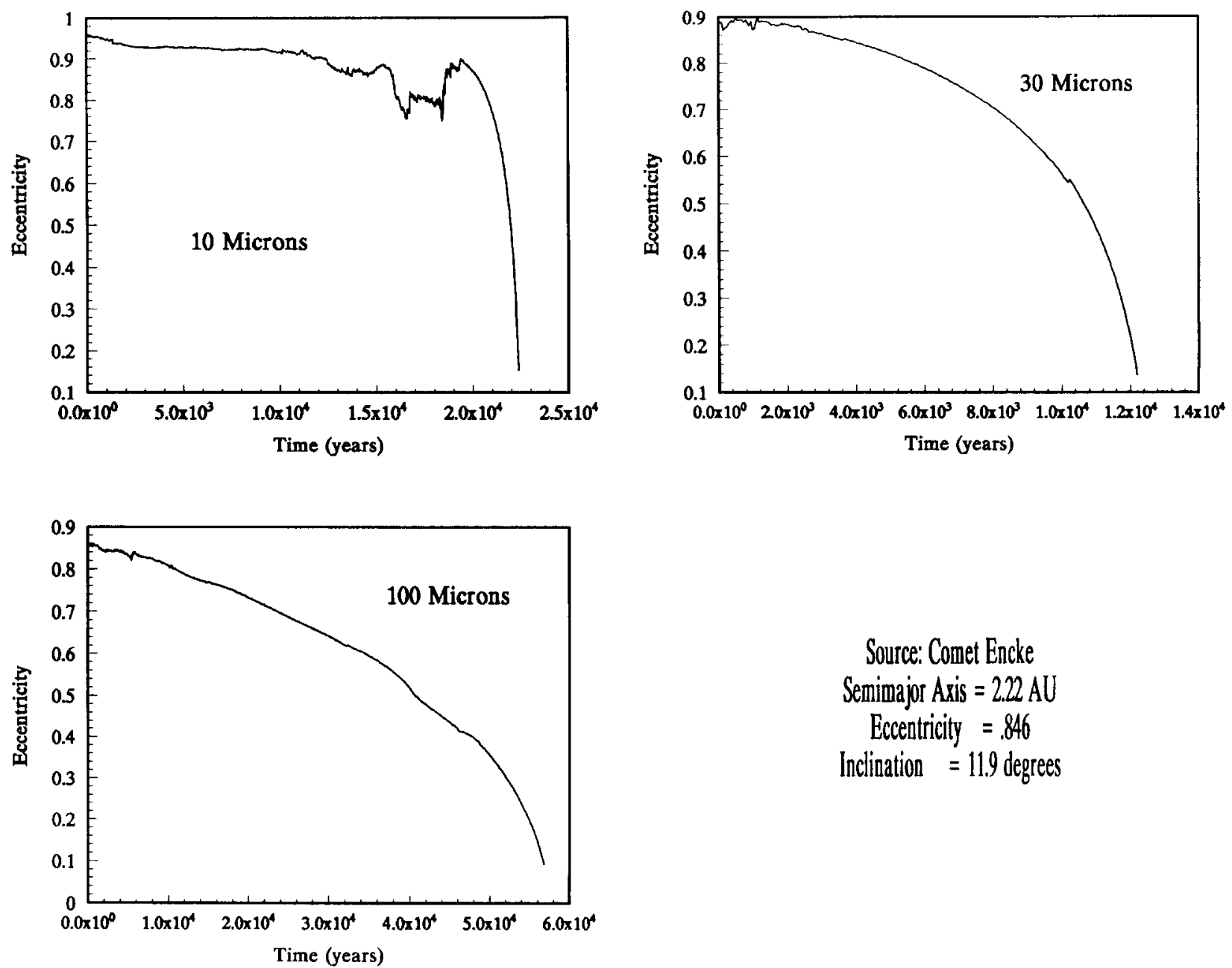

FIG. 9. Orbital eccentricity evolution of 10-, 30-, and 100- $\mu$ m-radius particles originating from Comet Encke.

aged that, when examined as an ensemble of evolved particle orbits, it will be possible to distinguish between comet- and asteroid-derived particles.

The "true" velocity distribution of cometary grains (if one could measure it) probably will be rather different from those shown in Figs. 15 and 16. This is because we did not include comets like Comet Halley, grains were only assumed to be released at zero velocity at perihelion, and grains that are now crossing the orbit of the Earth were doubtless often released 10,000 to 100,000 years ago from comets that may no longer exist. Nevertheless, we feel that we have shown that grains emitted from comets will generally be orbitally distinguishable, even after long intervals of orbital evolution, from grains emitted from comets.

We note one additional uncertainty that affects the orbital evolutionary history that we have developed, and that is the uncertainty in the magnitude of solar wind drag. Schwenn (1990), in an excellent review, shows that the solar wind momentum flux may vary somewhat with the solar sunspot cycle. Also the actual value of the solar wind momentum flux is probably uncertain to about the $10 \%$ level averaged over the 8 years that Schwenn analyzed, and may be yet more variable than that over very long time intervals. Finally, although the momentum flux remains about the same for the high speed $(>600 \mathrm{~km} / \mathrm{sec})$ solar wind as for the slow speed $(<400 \mathrm{~km} / \mathrm{sec})$ solar wind, the solar wind spatial density and, hence, the drag forces will be different for the two different solar wind velocity states. It is thought that at heliocentric latitudes greater than about $20 \mathrm{deg}$, the solar wind is almost all high speed. Changes in the solar wind drag will affect whether or not a grain makes a unique close passage by a planet or becomes trapped into a particular resonance with a planet. Instead, other close passages and trappings may occur. In this sense, the drag uncertainty mimics inaccuracies in the numerical integrator or approximations in the forces due to the planets.

We have not, in this paper, evaluated still more complicated scenarios such as one where a grain might be re- 

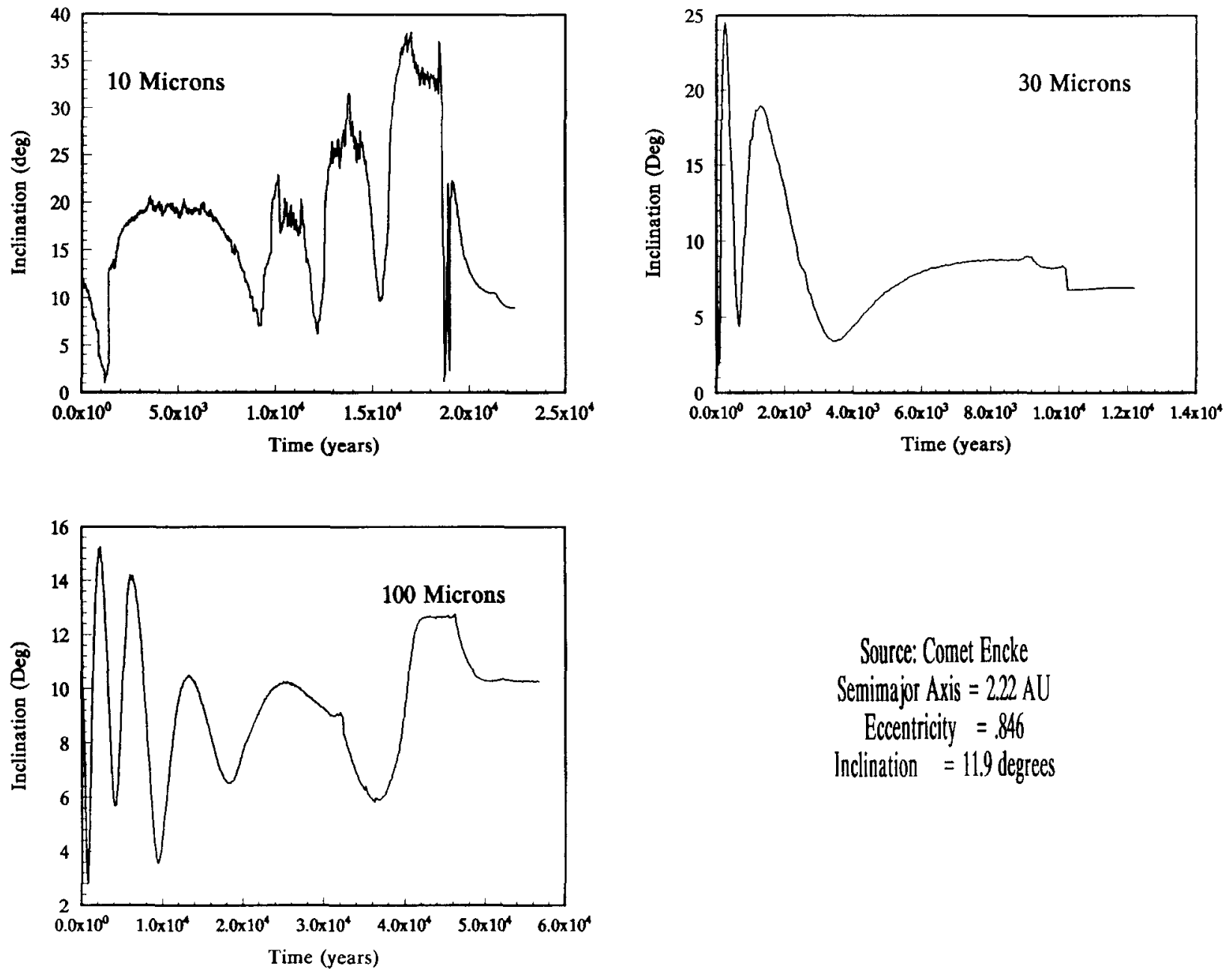

FIG. 10. Orbital inclination evolution of 10-, 30-, and 100- $\mu$ m-radius particles originating from Comet Encke

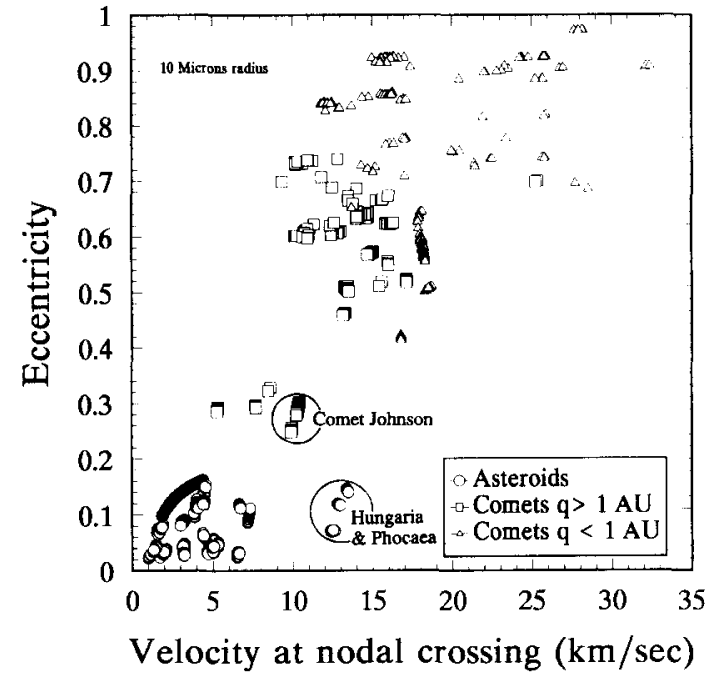

FIG. 11. Orbital eccentricity versus magnitude of relative velocity at nodal crossing with the Earth's orbit for $10-\mu \mathrm{m}$-radius particles derived from asteroids and comets.

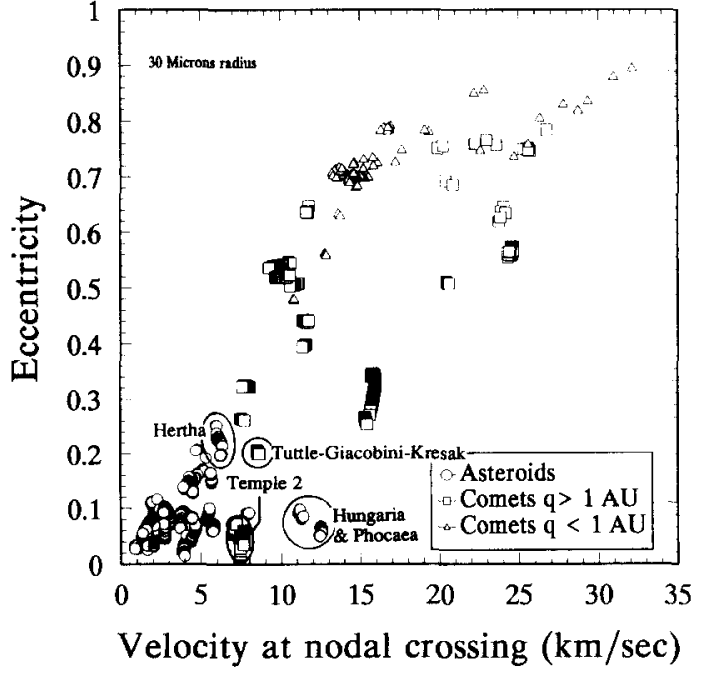

FIG. 12. Orbital eccentricity versus magnitude of relative velocity at nodal crossing with the Earth's orbit for 30- $\mu$ m-particles derived from asteroids and comets. 


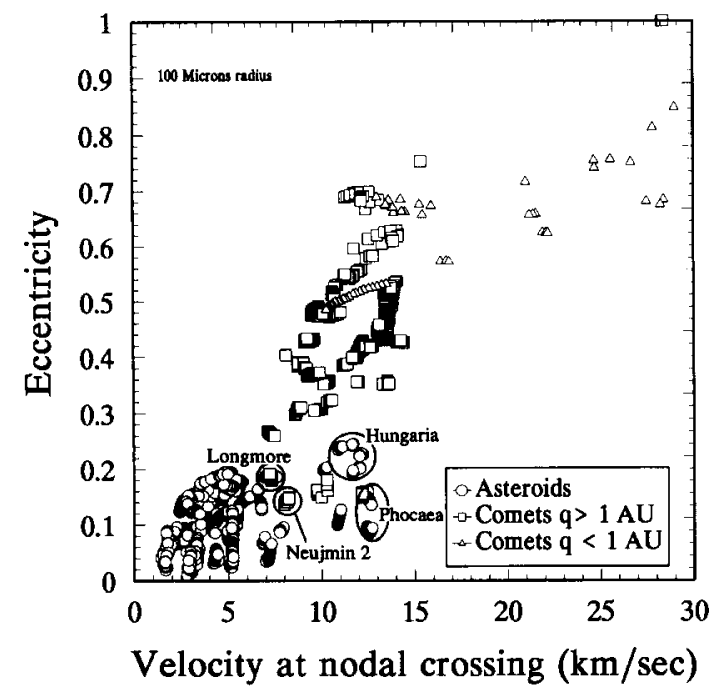

FIG. 13. Orbital eccentricity versus magnitude of relative velocity at nodal crossing with the Earth's orbit for $100-\mu \mathrm{m}$ particles derived from asteroids and comets.

leased from a parent body, differentially evolve away from the parent body, collide with another dust grain, and then permit the collision fragments to further orbitally evolve. When one compares collision lifetimes versus P-R drag lifetimes (Grün et al. 1985, Leinert and Grün 1990), one notes that they are about equal at particle masses of $10^{-5} \mathrm{~g}$. At masses only somewhat smaller than $10^{-5} \mathrm{~g}$, the particles usually do not suffer a collision before drifting all the way to the Sun where they are vaporized. Therefore the $10-\mu \mathrm{m}-$ and $30-\mu \mathrm{m}$-radius particles we simulated are unlikely to suffer catastrophic collisions, after release from their parent bodies, before they evolve in toward the Sun and are vaporized, or are ejected from the Solar

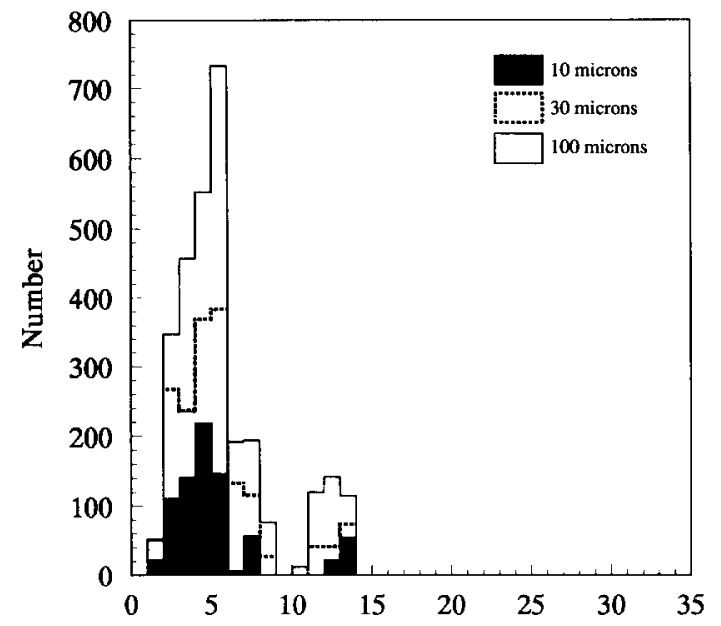

Magnitude of velocity at Nodal crossing, $\mathrm{km} / \mathrm{sec}$

FIG. 14. Histogram of magnitude of relative velocity at nodal crossing with the Earth for 10-, 30-, and $100-\mu \mathrm{m}$-radius particles from asteroids.

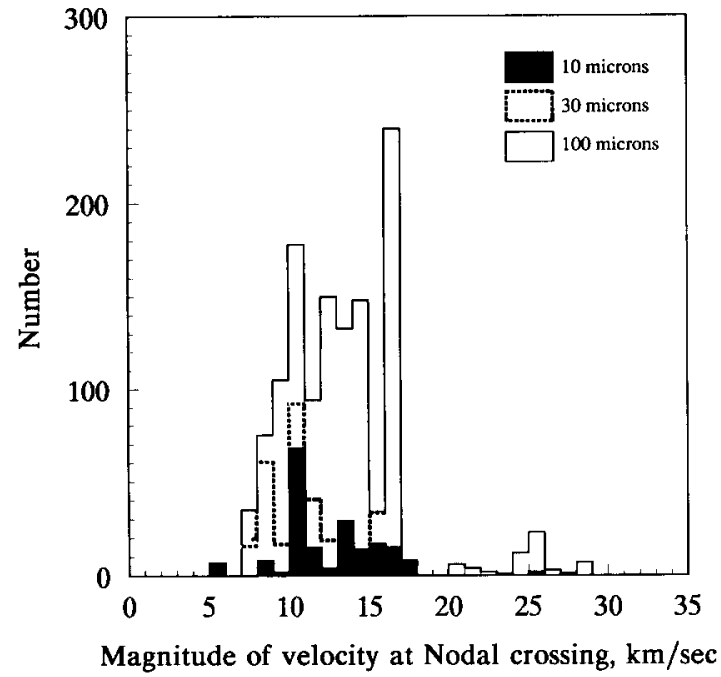

FIG. 15. Histogram of magnitude of relative velocity at nodal crossing with the Earth for 10-, 30-, and $100-\mu \mathrm{m}$-radius particles from comets with perihelion greater than $1 \mathrm{AU}$.

System. The $100-\mu \mathrm{m}$-radius particles, on the other hand, are likely to undergo a catastrophic collision before they are eliminated from the Solar System. Therefore our simulation of these latter grains is only true for that fraction that does not suffer catastrophic collisions after release from its parent bodies.

\section{CONCLUSIONS}

Even though dust particles follow very complicated orbital evolutionary histories, orbital eccentricities of asteroidal dust grains, as they pass the Earth's orbit, are lower than those of nearly all cometary dust grains (except

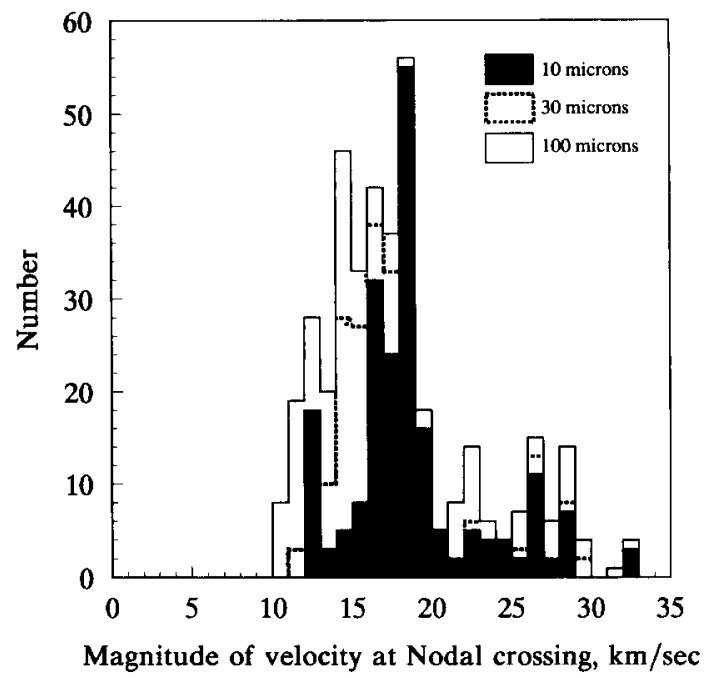

FIG. 16. Histogram of magnitude of relative velocity at nodal crossing with the Earth for 10-, 30-, and 100- $\mu$ m-radius particles from comets with perihelion less than $1 \mathrm{AU}$. 
for an occasional cometary dust grain that lands in the asteroidal grain $e$ versus $u$ field). Thus it appears that one may generally differentiate asteroidal grains from cometary grains on the basis of the measured orbital eccentricities alone.

The average values of eccentricity for the grains from asteroids of 10,30 , and $100 \mu \mathrm{m}$ radius are, respectively, $0.1,0.1,0.13$, and they cross the Earth's orbit with respective relative average velocities of 5,5 , and $6 \mathrm{~km} / \mathrm{sec}$. The corresponding respective eccentricities and relative velocities for the comets with $q>1 \mathrm{AU}$ are $0.5,0.4,0.4$, and 12,14 , and $12 \mathrm{~km} / \mathrm{sec}$; for comets with $q<1 \mathrm{AU}$, the respective values are $0.6,0.7,0.6$, and 15,16 , and 17 $\mathrm{km} / \mathrm{sec}$. We see that, even after many tens of thousands of years of orbital evolution, the orbits of cometary grains and asteroidal grains that we simulated do not, on the whole, resemble one another. Thus, it is very probable that broad parent-daughter associations can be made from measurements of their trajectories in Earth orbit.

\section{ACKNOWLEDGMENTS}

We thank Fred Hörz of JSC for his encouragement of these studies. We also thank B. Gustafson and an anonymous referee for their many constructive comments.

\section{REFERENCES}

Allen, C. W. 1962. Astrophysical Quantities, 2nd ed. The Athelone Press, University of London.

Burkhardt, G. 1985. Dynamics of dust particles in the Solar System. In Properties and Interactions of Interplanetary Dust (R. H. Giese and P. Lamy, Eds.), pp. 389-393. Reidel, Boston.

Burns, J. A., P. L. LAMY, ANd S. Soter 1979. Radiation forces on small particles in the Solar System. Icarus 40, 1-48.

Dermott, S. F.. P. D. Nicholson, J. A. Burns, and J. R. Houck 1984. Origin of the Solar System dust bands discovered by IRAS. Nature 312, 505-509.

Everhart, E. 1974. Implicit single-sequence methods for integrating orbits. Celest. Mech. 10, 35-55.

Everhart, E. 1985. An efficient integrator that uses Gauss-Radau spacings. In Dynamics of Comets: Their Origin and Evolution (A. Carusi and G. B. Valsecchi, Eds.), pp. 185-202. Reidel, Boston.

FlYNN, G. J. 1989. Atmospheric entry heating: A criterion to distinguish between asteroidal and cometary sources of interplanetary dust. Icarus 77, 287-310.

Gonzci, R., Ch. Froeschle, and C. Froeschle 1982. PoyntingRobertson drag and orbital resonance. Icarus 51, 633-654.

Gradie, J. C., C. R. Chapman, and J. G. Williams 1979. In Asteroids (T. Gehrels, Ed.), pp. 359-390. Univ. of Arizona Press, Tucson.

Gyün, E., H. A. Zook, H. Füchtig, AND R. H. Giese 1985. Collisional balance of the meteoritic complex. Icarus 62, 244-272.

Gustafson, B. Å. S., AND N. Y. Misconi 1986. Interplanetary dust dynamics. I. Long-term gravitational effects of the inner planets on zodiacal dust. Icarus 66, 280-287.

Gustafson, B. A.. S., N. Y. Misconi, ANd E. T. Rusk 1987a. Interplanetary dust dynamics. II. Poynting-Robertson drag and planetary perturbations on cometary dust. Icarus 72, 568-581.
Gustafson, B. A. S., N. Y. Misconi, and E. T. Rusk 1987b. Interplan etary dust dynamics. III. Dust released from P/Encke: Distribution with respect to the zodiacal cloud. Icarus 72, 582-592.

Hauser, M. G., F. C. Gillett, F. J. Low, T. N. Gautier, C. A. Beichman, G. Neugebauer, H. H. Auman,, B. Baud, N. BogGeSs, J. P. EMERSON, J. R. Houck, B. T. SOIFER, aNd R. G. Walker 1984. IRAS observations of the diffuse infrared background. Astrophys. J. 278, L15-L18.

Hörz, F., D. E. Brownlee, T. E. Bunch, D. Grounds, E. Grün, W. L. Quaide, Y. Rummel, and R. M. Walker 1990. Cosmic Dust Collection Facility: Scientific Objectives and Programmatic Relations (F. Horz, Ed.), NASA TM 102160.

JaCKSON, A. A., AND H. A. Zook 1989. A Solar System dust ring with the Earth as its shepherd. Nature 337, 629-631.

KRESAK, L. 1976. Orbital evolution of the dust streams released from comets. Bull. Astron. Inst. Czech. 27, 35-46.

LEINERT. C. AND E. GRüN 1990. Interplanetary dust. In Physics of the Inner Heliosphere: Large Scale Phenomenon (R. Schwen and E. Marsch, Eds.), pp. 204-275. Springer-Verlag, Berlin.

Low, F. J., D. A. Beintema, T. N. Gautier, F. C. Ghllett, C. A. Beichman. G. Neugebauer, E. Young, H. H. Aumann. N. Boggess, J. P. Emerson. H. J. Habing, M. G. Hauser, J. R. Houck, M. Rowan-Robinson, B. T. SOIFER, R. G. Wal.ker. and P. R. Wessetious 1984. Infrared cirrus: New components of the extended infrared emission. Astrophys. J. 278, L19-L22.

Marsden. B. 1989. Catalogue of Cometary Orbits. Minor Planet Center.

Papp, K. A., K. A. InNanen, and A. T. Patrick 1977. A comparison of five algorithms for numerical orbit computation in galaxy models. Celest. Mech. 18, 277-286.

Porter, J. G. 1963. Statistics of comet orbits. In The Moon. Meteorites and Comets (B. M. Middlehurst and G. P. Kuiper, Eds.), pp. 550-572. Univ. of Chicago Press, Chicago.

Press, W. H., B. P. Flannery, S. A. Teukolsky, and W. T. VetterI.ING 1986. Numerical recipes. In The Art of Scientific Computing. Cambridge Univ. Press, New York.

SCHWENN, R. 1990. Large scale structure of the interplanetary medium. In Physics of the Inner Heliosphere. I. Large Scale Phenomenon (R. Schwenn and E. Marsch. Eds.), pp. 99-181. Springer-Verlag. Berlin.

SyKes, M. V., AND R. Greenberg 1986. The formation and origin of the IRAS zodiacal dust bands as a consequence of single collisions between asteroids. Icaris $\mathbf{6 5}, 51-69$.

Sykes, M. V., L. A. Lebofsky, D. M. Hunten, and F. Low 1986. The discovery of dust trails in the orbits of periodic comets. Scicnce 232, $115-117$.

Sykes, M. V.. D. J. Lien, ANd R. G. Walker 1990. The Tempel 2 dust trail. Icarus 86, 236-247.

WHIPPI.E, F. L. 1967. On maintaining the meteoritic complex. In The Zodiacal Light and the Interplanetary Medium, NASA SP-150, pp. 409-426.

Wyatt, S. P., ANd F. L. WhipPle 1950. Poynting-Robertson effect on meteor orbits. Astrophys. J. 111, 134-141.

ZOOK, H. A. 1986. Precision requirements on cosmic dust trajectory measurements. In Trajectory Determinations and Collection of Micrometeoroids on the Space Station (F. Horz, Ed.), pp. 97-99. LPI Technical Report 86-05, Lunar and Planetary Institute, Houston.

Zook, H. A. 1988. On cosmic dust trajectory measurements and experiment pointing considerations. In Progress toward a Cosmic Dast Collection Facility on Space Station (I. D. R. Mackinnon and W. C. Carey, Eds.), pp. 76-77. LPI Technical Report 88-01, Lunar and Planetary Institute. Houston. 